EPJ Web of Conferences 108, 02025 (2016)

DOI: $10.1051 /$ epjconf/201610802025

(C) Owned by the authors, published by EDP Sciences, 2016

\title{
An Algorithm for the Simulation of the Magnetized Neutron Star Cooling
}

\author{
H. Grigorian 1,2,a , A. Ayriyan , E. Chubarian², A. Piloyan³ ${ }^{3}$ and M. Rafayelyan ${ }^{4}$ \\ ${ }^{1}$ Joint Institute for Nuclear Research, Joliot-Curie 6, 141980 Dubna, Moscow Region, Russia \\ ${ }^{2}$ Yerevan State University, Alek Manyukyan 1, 0025 Yerevan, Republic of Armenia \\ ${ }^{3}$ Yerevan Physics Institute, A. Alikhanian Brothers 2, 0036 Yerevan, Republic of Armenia \\ ${ }^{4}$ University of Bordeaux, 351 Cours de la Libération, F-33400 Talence, France
}

\begin{abstract}
A model and algorithm for the cooling of the magnetized neutron stars are presented. The cooling evolution described by a system of parabolic partial differential equations with non-linear coefficients is solved using the Alternating Direction Implicit method. A difference scheme and the preliminary results of simulations are presented.
\end{abstract}

\section{Introduction}

Modelling of magnetized neutron stars cooling process is an interesting subject for the investigations of the internal structure of such objects. The main aspect is the investigation of the nuclear matter properties at extremely high densities. The magnetic field of compact object when it is more than $10^{14} \mathrm{G}$ has a significant influence on the heat transport inside the star and can give observational effects on the surface temperatures. The model of cooling evolution is given by a system of parabolic partial differential equations with non-linear temperature depending thermal coefficients and cooling (via neutrinos) and heating (due to magnet field decay) sources.

Due to the axial symmetry of the field and spherical symmetry of the matter distribution inside, the problem can be described using spherical coordinates in spatial 2D. For the solution we choose the Alternating Direction Implicit (ADI) method $[1,2]$. In the difference scheme we use non-constant spatial steps and self-correcting time

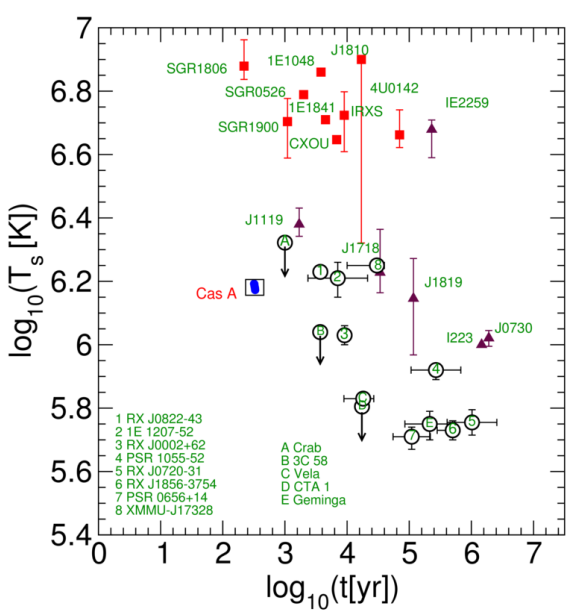

Figure 1: Observational data of age and temperature of compact stars. Squares - AXPs and SGRs neutron stars with magnetic field from $10^{14} \mathrm{G}$ up to $10^{15} \mathrm{G}$. Triangles - radioquiet stars with field around $10^{13} \mathrm{G}$. Circles radio-pulsars with field $\leq 10^{12} \mathrm{G}$.

a e-mail: hovikgrigorian@gmail.com 
step. We investigate the special boundary conditions in the center and on the surface of the star configuration. At the current stage of the study we are going to present some preliminary results of the cooling simulations to demonstrate the efficiency of our 2D cooling algorithm.

The nowadays observational data of the magnetized neutron star (Fig. 1, for the table of data with corresponding references, see [3]) could not be explained by 1D cooling simulations neglecting the magnetic field inside. The inclusion of the magnetic field is necessary but not sufficient yet [3, 4]. In this work we focus on an algorithm of 2D simulation of the cooling of a magnetized neutron star.

\section{Equations for Thermal Evolution of the Magnetized Compact Star}

The neutron star is surrounded by a gravitational field which, in the approximation of slow rotation of the stars, can be described by the metric tensor of the space-time manifold [5]

$$
d s^{2}=-e^{2 \Phi} d t^{2}+e^{2 \Lambda} d r^{2}+r^{2} d \Omega^{2} .
$$

The compact star configuration is constructed with the use of the equation of state (EoS) of the stellar matter based on the knowledge of the nuclear matter [6]. The coefficients of the metric tensor as well as characteristics of the matter distribution are self-consistent solutions of the Einstein equation (specially for this case TOV $[5,6]$ ) where the temperature effect on the internal structure could be neglected because of the high density inside the star.

On the other hand the thermal evolution of the star can be described using the energy balance equation, which has parabolic form:

$$
c_{v} e^{\Phi} \frac{\partial T}{\partial t} \quad+\vec{\nabla} \cdot\left(e^{2 \Phi} \vec{F}\right)=e^{2 \Phi} Q
$$

where $c_{v}$ is the specific heat per unit volume and $Q$ is the energy loss/gain by neutrino emission, Joule heating, accretion heating, etc. The vector $\vec{F}$ corresponds to the heat flux.

The thermal properties of the stellar matter are investigated under of different nuclear matter cooling scenarios [7-11].

The flux is created due to the temperature gradient and tends to equilibrate the temperature

$$
\vec{F}=-e^{-\Phi} \hat{\kappa} \cdot \vec{\nabla}\left(e^{\Phi} T\right) .
$$

Here $\hat{\kappa}$ is the thermal conductivity, which is a tensor under an enough strong magnetic field of the star.

Hereafter we will use the notations $\tilde{T} \equiv e^{\Phi} T$ and $\tilde{F} \equiv e^{2 \Phi} F$. The flux components (in spherical coordinates) of the dipole magnetic field are:

$$
\tilde{F}_{r}=-e^{\Phi}\left(\kappa_{r r} e^{-\Lambda} \partial_{r} \tilde{T}+\frac{\kappa_{r \theta}}{r} \partial_{\theta} \tilde{T}\right), \quad \tilde{F}_{\theta}=-e^{\Phi}\left(\kappa_{\theta r} e^{-\Lambda} \partial_{r} \tilde{T}+\frac{\kappa_{\theta \theta}}{r} \partial_{\theta} \tilde{T}\right),
$$

with the $\phi$-component vanishing identically due to the axial symmetry.

The magnetic field, which affects the conductivity, leads to anisotropy along and orthogonal to the field. The relation between the conductivity components is defined in terms of the magnetization parameter $\omega_{B} \tau$ :

$$
\frac{\kappa_{\|}}{\kappa_{\perp}}=1+\left(\omega_{B} \tau\right)^{2},
$$

where $\tau$ is the particle relaxation time [12], and $\omega_{B}$ is the classical gyrofrequency corresponding to the magnetic field strength $B$,

$$
\omega_{B}=\frac{e B}{m c},
$$


$m$ is the effective mass, $e$ is the charge of the particle in the magnetic field, and $c$ is the speed of light.

Using the unit vector $\vec{b}$ of the magnetic dipole the flux, we get:

$$
\vec{F}=-e^{\Phi} \kappa_{\perp}\left[\vec{\nabla} \tilde{T}+\left(\omega_{B} \tau\right)^{2}(\vec{b} \cdot \vec{\nabla} \tilde{T}) \cdot \vec{b}+\omega_{B} \tau(\vec{b} \times \vec{\nabla} \tilde{T})\right] .
$$

When the magnetic field is parallel to the axis $z$ one has $b=(\cos \theta,-\sin \theta, 0)$. So for the components of the conductivity we get:

$$
\begin{aligned}
\kappa_{r r} & =\frac{1+\left(\omega_{B} \tau\right)^{2} \xi^{2}}{1+\left(\omega_{B} \tau\right)^{2}} \kappa_{\|}, \\
\kappa_{\theta r} & =\kappa_{r \theta}=\xi \sqrt{1-\xi^{2}}\left(\kappa_{\|}-\kappa_{\perp}\right), \\
\kappa_{\theta \theta} & =\frac{1+\left(\omega_{B} \tau\right)^{2}\left(1-\xi^{2}\right)}{1+\left(\omega_{B} \tau\right)^{2}} \kappa_{\|} .
\end{aligned}
$$

For simplicity we will use the following notations: $\xi \equiv \cos (\theta), \tilde{Q} \equiv e^{2 \Phi} Q$.

The introduction of the new variable $\xi$ and of the accumulated mass $m=4 \pi \int \rho r^{2} d r$ (here $\rho$ is the energy density of the matter) instead of $\theta$ and $r$ transforms the thermal evolution equation (2) to the following form

$$
\frac{\partial u}{\partial t}=D_{1} \frac{\partial}{\partial m}\left(B_{1 m} \frac{\partial u}{\partial m}+B_{1 \xi} \frac{\partial u}{\partial \xi}\right)+D_{2} \frac{\partial}{\partial \xi}\left(B_{2 m} \frac{\partial u}{\partial m}+B_{2 \xi} \frac{\partial u}{\partial \xi}\right)+Q_{B}
$$

The sought-for function is $u=\log (\tilde{T})$ and the coefficients are

$$
\begin{gathered}
B_{1 m} \equiv 4 \pi r^{4} \rho e^{-\Lambda}\left(1+\left(\omega_{B} \tau\right) \xi^{2}\right) k_{\perp} \tilde{T} e^{\Phi}, \quad B_{2 m} \equiv 4 \pi r \rho e^{-\Lambda} \xi\left(1-\xi^{2}\left(\omega_{B} \tau\right)^{2} k_{\perp} \tilde{T} e^{\Phi},\right. \\
B_{1 \xi} \equiv r \xi\left(1-\xi^{2}\right)\left(\omega_{B} \tau\right)^{2} k_{\perp} \tilde{T} e^{\Phi}, \quad B_{2 \xi} \equiv\left(1+\left(\omega_{B} \tau\right)^{2}\left(1-\xi^{2}\right)\right)\left(1-\xi^{2}\right) k_{\perp} \tilde{T} e^{\Phi} / r^{2},
\end{gathered}
$$

and $D_{1} \equiv 4 \pi D_{2} \rho e^{-\Lambda}, D_{2} \equiv\left(c_{v} \widetilde{T}\right)^{-1}, Q_{B} \equiv D_{2} \tilde{Q}$.

\section{2D Scheme for Mixed Implicit and Explicit Methods}

Numerical calculations were done using the most convenient ADI method [1, 2] from the point of view of the stability. The discretization with respect to $m$ was done taking into account the energy distribution $\rho(r)$. This function is the solution of a single compact star for a given central density or a given total mass. At each given distance $r_{j}$ from the center, we have the grid points $m_{j}$. The mass at a given $j$ is the same for all angles, therefore $\xi$ could be considered as an independent coordinate.

The steps of discretization are $\Delta m_{j \pm 1 / 2}= \pm\left(m_{j \pm 1}-m_{j}\right)$ and $\Delta m_{j}=(1 / 2)\left(\Delta m_{j+1 / 2}+\Delta m_{j-1 / 2}\right)=$ $(1 / 2)\left(m_{j+1}-m_{j-1}\right)$. Similar notations are used for the angle $\xi$ as well as for any function $F(r, \theta)$ : $F_{i, j \pm 1 / 2}=(1 / 2)\left(F_{i, j \pm 1}+F_{i, j}\right)$.

The first order partial derivatives have been approximate by first order finite differences,

$$
\left(\frac{\partial u}{\partial m}\right)_{i, j \pm 1 / 2}= \pm \frac{u_{i, j \pm 1}-u_{i j}}{\Delta m_{j \pm 1 / 2}}, \quad\left(\frac{\partial u}{\partial \xi}\right)_{i \pm 1 / 2, j}= \pm \frac{u_{i \pm 1, j}-u_{i j}}{\Delta \xi_{i \pm 1 / 2}} .
$$

For the second order derivatives, the $R$ and $S$ operators corresponding to a same or to mixed variables were defined,

$$
\left[R^{(1)} u\right]_{i j} \equiv-D_{1} \frac{\partial}{\partial m}\left(B_{1 m} \frac{\partial u}{\partial m}\right)_{i, j}=-\frac{D_{1 i, j}}{\Delta m_{j}}\left(B_{1 m, i, j+1 / 2} \frac{u_{i, j+1}-u_{i, j}}{\Delta m_{j+1 / 2}}-B_{1 m, i, j-1 / 2} \frac{u_{i j}-u_{i j-1}}{\Delta m_{j-1 / 2}}\right),
$$




$$
\left[R^{(2)} u\right]_{i j} \equiv-D_{2} \frac{\partial}{\partial \xi}\left(B_{2 \xi} \frac{\partial u}{\partial \xi}\right)_{i, j}=-\frac{D_{2 i, j}}{\triangle \xi_{i}}\left(B_{2 \xi, i+1 / 2,, j} \frac{u_{i+1, j}-u_{i, j}}{\triangle \xi_{i+1 / 2}}-B_{2 \xi i-1 / 2, j} \frac{u_{i j}-u_{i-1, j}}{\triangle \xi_{i-1 / 2}}\right)
$$

and

$$
\begin{gathered}
{\left[S^{(1)} u\right]_{i j}=D_{1} \frac{\partial}{\partial m}\left(B_{1 \xi} \frac{\partial u}{\partial \xi}\right)_{i j}=\frac{D_{1 i, j}}{2 \triangle m_{j}}\left(B_{1 \xi, i, j+1} \frac{u_{i+1, j+1}-u_{i-1, j+1}}{2 \triangle \xi_{i}}-B_{1 \xi, i, j-1} \frac{u_{i+1 j-1}-u_{i-1, j-1}}{2 \triangle \xi_{i}}\right),} \\
{\left[S^{(2)} u\right]_{i j}=D_{2} \frac{\partial}{\partial \xi}\left(B_{2 m} \frac{\partial u}{\partial m}\right)_{i, j}=\frac{D_{2 i, j}}{2 \triangle \xi_{i}}\left(B_{2 m, i+1, j} \frac{u_{i+1, j+1}-u_{i+1, j-1}}{2 \triangle m_{j}}-B_{2 m, i-1, j} \frac{u_{i-1 j+1}-u_{i-1, j-1}}{2 \triangle m_{j}}\right) .}
\end{gathered}
$$

After discretization of the time with the steps $\Delta t_{n}$, the differential equation of the problem can be written as:

$$
\frac{u^{n+1}-u^{n}}{\triangle t_{n}}+\sigma R^{(1)} u^{n+1}+(\sigma-1) R^{(2)} u^{n+1}=(\sigma-1) R^{(1)} u^{n}-\sigma R^{(2)} u^{n}+S^{(1)} u^{n}+S^{(2)} u^{n}+Q_{B} .
$$

The introduced parameter $\sigma$ stands for the ADI method: at $\sigma=1$ the implicit method is used with respect to $m$, while at $\sigma=0$, with respect to $\xi$.

The non-vanishing elements in the right hand side of this algebraic equation can be written in the following form

$$
\begin{aligned}
X^{[i][j]} & =\sigma \frac{K_{i, j+1 / 2}^{(1)}}{\left(\Delta m_{j}\right)^{2}}+(\sigma-1) \frac{K_{i+1 / 2, j}^{(2)}}{\left(\Delta \xi_{i}\right)^{2}} \\
Z^{[i][j]} & =\sigma \frac{K_{i, j-1 / 2}^{(1)}}{\left(\Delta m_{j}\right)^{2}}+(\sigma-1) \frac{K_{i-1 / 2, j}^{(2)}}{\left(\Delta \xi_{i}\right)^{2}} \\
Y^{[i][j]} & =1 / \Delta t_{n}-X^{[i][j]}-Z^{[i][j]}, \\
S^{[i][j]} & =u_{i j}^{n} / \Delta t_{n}+(\sigma-1)\left[R^{(1)} u^{n}\right]_{i j}-\sigma\left[R^{(2)} u^{n}\right]_{i j}+\left[S^{(1)} u^{n}\right]_{i j}+\left[S^{(2)} u^{n}\right]_{i j}+\left[Q_{B}\right]_{i j},
\end{aligned}
$$

where we use the following notations: $X^{[i][j]}$ and $Z^{[i][j]}$ are upper and lower diagonal vectors correspondingly, $Y^{[i][j]}$ is the main diagonal vector. For solving the obtained tri-diagonal algebraic system, the Thomas algorithm is used [13, 14]. values

In (6), the $K_{i, j}^{(1)}$ and $K_{i, j}^{(2)}$ are the centred mean

$$
\begin{gathered}
K_{i, j \pm 1 / 2}^{(1)}=D_{1 i, j} B_{1 m i, j \pm 1 / 2} \frac{\Delta m_{j}}{\Delta m_{j \pm 1 / 2}}, \\
K_{i \pm 1 / 2, j}^{(2)}=D_{2 i, j} B_{2 \xi i \pm 1 / 2, j} \frac{\Delta \xi_{j}}{\Delta \xi_{j \pm 1 / 2}} .
\end{gathered}
$$

To complete the tri-diagonal algebraic system, a set of boundary conditions obeying to the physical requirements, at the center and on the surface of the neutron star is defined as follows [15]. All fluxes of heat from all directions have to vanish at the center. This leads to $X^{[i][0]}=0$. Therefore, the temperature at the center point could be set the mean value of temperatures in a close neighbourhood of the center.

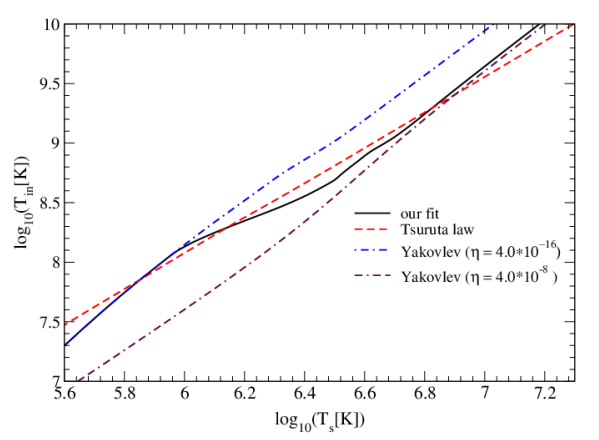

Figure 2: Relation between the mantle temperature $T_{\mathrm{m}}$ and the surface temperature $T_{\mathrm{s}}$ 
At the surface we assume that the flux of the energy along the radial direction has to equate the flux of energy of the photon emission:

$$
L_{m}(i, J)=2 \pi R^{2} \sigma \tilde{T}_{s}^{4}(i) \Delta \xi_{i},
$$

where $J$ is the maximal value of the index $j, \sigma$ is the Boltzmann constant and $T_{s}$ is the surface temperature, which is connected to $T_{m}$, the mantle temperature under the core (Fig. 2):

$$
u_{i, J}=\log \tilde{T}_{m}(i)
$$

A detailed discussion of this point was reported in [7].

From symmetry reasons, the tangential fluxes on the polar and equatorial planes also are zero $L_{\xi}(i=0, j)=L_{\xi}(i=I, j)=0$ :

$$
L_{\xi}(i, j)=\left(B_{1 \xi} \frac{\partial u}{\partial \xi}\right)_{i, j}=B_{1 \xi, i, j} \frac{u_{i+1, j}-u_{i-1, j}}{2 \Delta \xi_{i}} .
$$

\section{Some Features of the Simulations and Conclusions}

To demonstrate the work of our algorithm we provide simulations with model coefficients, which are taking into the account the main features of the physical properties of the thermal coefficients. Particularly, we use the cooling mechanism of the neutrino emission, the so called modified URCA process (see for details [16]). We also vary the magnetic field, however we don't include the Joule heating from the decay of magnetic field.

As it can be seen from the Fig. 3a, due to absence of additional anisotropic heating sources, the temperature distribution in all directions becomes isotropic after 50 years of evolution. And we can notice as well that this property is independent of the value of the initial anisotropic distribution of the temperature.

One can notice that, if the temperature along some direction is initially smaller then the temperature at the beginning of the isotropic era it heats up during the evolution (see dotted lines for the lowest arrow in Fig. 3a). It means that the heat conducting process dominates the neutrino cooling.

We notice that a period of $50-100$ years is short enough to be able to compare this results with observations, because all available data (Fig. 1) are for objects older than at least 500 years. On the other hand one can see that the temperature values at the beginning of the isotropic era are around the same what are the observational temperatures of already cold magnetars.

It means that the heat retention inside the star for such a long time could not be just the effect of the anisotropy due to the magnetization. It is even hard to argue that the observed temperatures of red point in Fig. 1 are estimated only for small area around the poles of the stars.

In the Fig. $3 b$ we demonstrate the automatic choice of the time-step of the numerical algorithm. The top line on this figure is the predicted time-step, the bottom curve is the modified time-step to provide stability of the algorithm. The automatic choice of the time-step is needed mainly due to the non-trivial mass distribution inside the star.

\section{Acknowledgements}

The study is partially supported by RFBR according to the scientific project No. 14-01-00628. Authors thank Dr. Edik Ayryan (LIT, JINR) for fruitful discussions and supporting the participation of HG on MMCP'2015. HG, AA, MR acknowledge Ter-Antonyan-Smorodinskii Program for supporting exchange between JINR Dubna and Armenian Universities and Institutes. HG, EC, AP and MR thank Ministry of Education and Science of Armenia for the support according to the scientific project of State Committee of Science, grant No. H-11-1c259. 


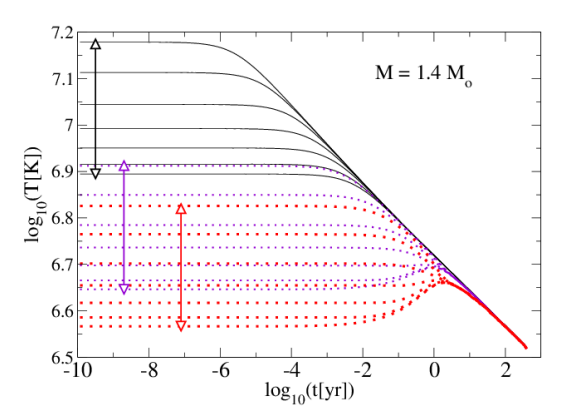

(a) Surface temperature evolution of neutron star with mass $m=1.4_{\text {sun }}$. Each color corresponds to cooling curves for different azimuthal angles of one object.

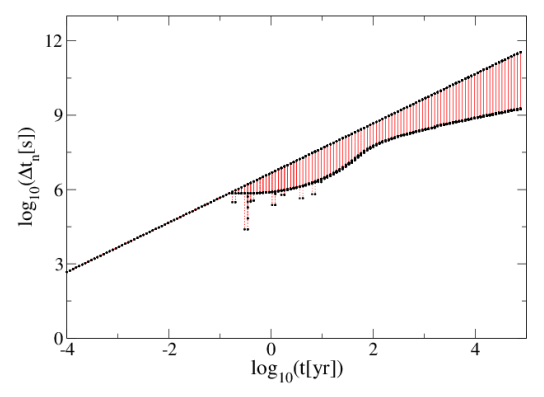

(b) The choice of the time-step during the numerical simulation.

Figure 3: Simulation results of the coolings of magnetars

\section{References}

[1] A. Samarskii and P. Vabishchevich, Computational Heat Transfer, Volume 1 (John Wiley \& Sons Ltd., Chichester, England, 1995)

[2] N. Yanenko, Fractional step methods for solution of multidimensional problems of mathematical physics (Nauka, Moscow, 1967) (in russian)

[3] D. Aguilera, J. Pons, and J. Miralles, The Astroph. J. Lett. 673, 2, L167-L170 (2008)

[4] D.N. Aguilera, J.A. Pons, and J.A. Miralles, Astron. \& Astroph. 486, 255-271 (2008)

[5] C. Misner, K. Thorne, and J. Wheeler, Gravitation (W.H. Freeman and Co., San Francisco, 1973)

[6] F. Weber, Pulsars as Astrophysical Laboratories for Nuclear and Particle Physics (IOP Publishing, Bristol, Great Britain, 1999)

[7] D. Blaschke, H. Grigorian, and D.N. Voskresensky, Astron. \& Astroph. 424, 979-992 (2004)

[8] H. Grigorian and D.N. Voskresensky, Astron. \& Astroph. 444, 3, 913-929 (2005)

[9] H. Grigorian, D. Blaschke, and D. Voskresensky, Phys. Rev. C 71, 045801 (2005)

[10] D. Page, J. Lattimer, M. Prakash, and A. Steiner, The Astroph. J. Suppl. Ser. 155, 623 (2004)

[11] D. Page, J. Lattimer, M. Prakash, and A. Steiner, The Astroph. J. 707, 2 (2009)

[12] V. Urpin and D. Yakovlev, Soviet Astronomy 24, 425 (1980)

[13] L. Thomas, Elliptic Problems in Linear Differential Equations over a Network (Watson Sci. Comput. Lab Report, Columbia University, New York, 1949)

[14] W. Press, S. Teukolsky, W. Vetterling, and B. Flannery, Numerical Recipes, third ed. (Cambridge University Press, New York, 2007)

[15] D. Yakovlev, K. Levenfish, A. Potekhin, O. Gnedin, and G. Chabrier, Astron. \& Astroph. 417, 169-179 (2004)

[16] H. Grigorian, EPJ Web Conf. 7, 03004 (2010) 